\title{
Is there enough room for non-invasive ventilation in pulmonary rehabilitation?
}

\section{Laura D Ciobanu}

Associate Professor, Department of Internal Medicine, University of Medicine and Pharmacy, 700115 Iasi, Romania.

Corresponding Author: Laura D Ciobanu, Associate Professor, Department of Internal Medicine, University of Medicine and Pharmacy, Grigore T Popa, Iasi; 700115 Iasi, Romania.

Received date: October 26, 2020; Accepted date: November 28, 2020; Published date: December 02,2020

Citation: Laura D Ciobanu, (2020) Is there enough room for non-invasive ventilation in pulmonary rehabilitation? J, Biotech. and Bioproc. 1(2); DOI: $10.31579 / 2766-2314 / 007$

Copyright: ( ) 2020, Laura D Ciobanu, This is an open access article distributed under the Creative Commons Attribution License, which permits unrestricted use, distribution, and reproduction in any medium, provided the original work is properly cited.

\begin{abstract}
Pulmonary rehabilitation (PR) is a non-pharmacological intervention addressed to chronic obstructive pulmonary disease (COPD) and non-COPD chronic respiratory patients, a key management strategy scientifically demonstrated to improve exercise capacity, dyspnoea, health status and psychological wellbeing. The main body of literature comes from COPD patients, as they provide the core evidence for PR programmes. PR is recommended even to severe patients having chronic respiratory failure; their significant psychological impairment and potential for greater instability during the PR programme will be carefully considered by the multidisciplinary team. Optimizing medical management (eg, inhaled bronchodilators, oxygen therapy, non-invasive ventilation) may enhance the results of exercise training. Patients who already receive long-term domiciliary non-invasive ventilation (NIV) for chronic respiratory failure might exercise with NIV during exercise training if acceptable and tolerable to the patient. It is not advisable to offer long-term domiciliary NIV with the only aim to improve outcomes during PR course. There are different attempts to use both negative and positive NIV in limited clinical studies. Long-term adherence to exercise is an important goal of PR programmes and teams, targeting to translate all-domain gains of PR into increased physical activity and participation to real life. Being a reliable alternative for the future, studies should focus on pressure regimens, type of devices, acceptability and portability for everyday activities.
\end{abstract}

Key words: chronic obstructive pulmonary disease, pulmonary rehabilitation, non-invasive ventilation

\section{Abbreviations}

AECOPD - acute exacerbation of chronic obstructive pulmonary disease

BiPAP - bilevel positive airway pressure

COPD - chronic obstructive pulmonary disease

CPAP - continuous positive airway pressure

CHRF - chronic hypercapnic respiratory failure

EPAP - expiratory positive airway pressure

FEV1 - forced expiratory volume in 1 second

GOLD - The Global Initiative for Chronic Obstructive Lung Disease

HOT-HMV - home oxygen therapy and home mechanical ventilation

HMV - home mechanical ventilation

HRQoL - health-related quality of life

ICU - intensive care unit

LTOT - long-term oxygen therapy

MIE - mechanical insufflation-exsufflation

6MWT - six-minute walking test

NIV - non-invasive ventilation

NPPV - non-invasive positive pressure ventilation
NPV - negative pressure ventilation

PaCO2 - arterial partial pressure of carbon dioxide

PAP - positive airway pressure

PAV - proportional assist ventilation

pNIV - portable non-invasive ventilation

PR - pulmonary rehabilitation

SpO2\% - saturation of arterial blood with oxygen measured by pulse oximetry

\section{The concept of pulmonary rehabilitation briefly}

Pulmonary rehabilitation is described as a "comprehensive intervention based on a thorough patient assessment followed by patient-tailored therapies that include, but are not limited to, exercise training, education, and behaviour change, designed to improve the physical and psychological condition of people with chronic respiratory disease, and to promote the long-term adherence to health-enhancing behaviours" [1]. The main goal for PR programmes is to enhance physical activity towards normal levels, to return the patient to the highest possible capacity in order to achieve the maximum level of independence and functioning in the community $[2,3]$. Despite being a cost-beneficial intervention, only approximately two-fifth of chronic respiratory patients have been informed by their health care provider about PR and its positive results. 
This might be an explanation why $<2 \%$ of the patients are referred to one of the available PR programmes [4]. The main body of evidences are coming from the COPD patients, but PR is effective as well in many others obstructive and restrictive conditions.

COPD is a leading cause of morbidity and mortality, with an increased burden of disease worldwide and constitutes a major healthcare concern $[5,6]$. COPD patients are referred to PR due to persistent respiratory symptoms and/or limited activities of daily living and an unsatisfactory response to medical treatment offered in primary care [7]. PR addresses breathlessness, the perceived discomfort of breathing, a common symptom too many respiratory and systemic diseases [8]. Breathlessness is the consequence of imbalance between the increased respiratory muscle load and reduced ventilatory capacity [8]. Neural respiratory drive, the electrical output from the brainstem to the respiratory muscles, increases in response to this imbalance and acts to maintain an appropriate ventilation, thus becoming a major contributor to the subjective breathlessness [8, 9]. Breathlessness occurs in COPD, with disease advancement or in AECOPD as a result of imbalance in the load-capacitydrive relationship of the ventilatory system [8], with dynamic hyperinflation and impaired gas exchange that worsen ventilationperfusion mismatch [10].

COPD is known to induce, apart from respiratory symptoms, a decrease in muscle strength and endurance due to systemic inflammation, vulnerability to fatigue and a decline in exercise capacity and cardiac function [9, 11, 12, and 13]. A decrease in physical activity and the consecutive sedentary life, along to the weakened pulmonary function, will result in a vicious cycle with decline in health-related quality of life (HRQoL) and physical ability at a disproportionate rate comparing to the decline of lung function [11]. Therefore, COPD has extensively been reported as a complex disease affecting patients' health beyond the lungs with multiple intrapulmonary and extra pulmonary components and considerable variability between individuals [2].

Multidisciplinary PR is a key component in the management of COPD [5], and has proved to be beneficial in patients with COPD in terms of improving exercise capacity, symptoms (as breathlessness, fatigue, and mood) and HRQoL $[14,15]$; it reduces health care utilization, being one of the most cost-effective therapeutic strategies [5, 12]. PR is addressed to stable patients especially with moderate-to-severe disease, after an acute exacerbation, in intensive care unit, in perioperative period after a lung transplantation, before and after lung cancer surgery, and before endobronchial lung volume reduction [12]. It can be offered in a hospitalbased outpatient setting, in an inpatient setting, a community-based setting and at the patient's home [4].

Large differences in results may arise from exercise type, level of supervision, education and physiotherapy strategies, psychological support, use of medication and mostly of duration and frequency of the maintenance programmes [14]. The longer the duration of the PR programmes the greater sustained benefits in comparison with the shorter ones [14]. A contribution may have the patients' adherence to the programme, severity of disease, comorbidities, and accessibility of the PR premises [14]. These benefits tend to wane over time and most measures of improvement return to baseline by 12-24 months [5, 14]. Therefore, experts recommend continuation of exercise training beyond initial PR in order to prevent a decline in exercise capacity [14]. Maintenance programmes may consist in simple techniques used in ambulatory, community, or home programmes [14]. Home-based exercise interventions are safe and beneficial, helpful for patients who lack access to or are unable to participate in centre-based PR programmes; the increase on PA derives from factors around the patients, like an active spouse, walking the dog and other pets, and grand parenting [16].
Exercise training is the cornerstone in PR programmes and the best approach for increasing muscle strength, decreasing symptoms, reducing mood abnormalities, improving cardiovascular function and the motivation for physical activity [12]. The target training intensity in the PR exercise setting is critically dependent on baseline exercise testing, which is associated with a learning effect [16]. The main components of exercise training programmes are endurance and resistance training that should be practised supervised at least twice weekly, more than $60 \%$ to $80 \%$ of the maximal work rate, delivered as high-intensity and dynamic, interval and continuous training $[9,12,13,17]$; in parallel with these supervised sessions, there are recommended five unsupervised sessions of $30 \mathrm{~min}$ of PA per week, in line with standard healthy living advice [17]. Typical modes of aerobic exercise are walking or cycling [13]. In stable COPD patients, a combination of endurance and resistance training should be performed to maximize improvement in limb muscle function and whole-body exercise capacity [9].

\section{Non-invasive ventilation or why to think about it}

In advanced stages of respiratory disease, patients frequently develop chronic hypercapnic respiratory failure (CHRF). NIV is the standard treatment for patients with $\mathrm{CHRF}$ due to COPD and restrictive lung diseases, and a major indication for home mechanical ventilation (HMV) in Europe [6]. COPD patients benefit from NIV once they have COPD GOLD stage III or IV and CHRF $(\mathrm{PaCO} 2>6.0 \mathrm{kPa})$ in a stable clinical condition. Recent pulmonary rehabilitation BTS guidelines [17] suggest that NIV during exercise training should be offered to patients who already receive domiciliary NIV. With increased use of high-pressure NIV for home therapy, the use of NIV during PR would become more feasible.

In patients with chronic hypercapnic respiratory failure, long-term noninvasive positive pressure ventilation (NPPV) improves important physiological variables such as blood gases and lung hyperinflation. Results from clinical studies have shown that NPPV improves exercise capacity, exercise-related dyspnoea, pulmonary cachexia, sleep quality and QRQoL. Moreover, NPPV treatment might be associated with fewer hospital admission and lower overall treatment costs [18]. The best results with long-term NPPV have been noticed in studies using more intensive forms of NPPV, with higher inspiratory pressures and high back-up frequencies that have improved or even normalised hypercapnia $[6,18$, 19, and 20].

Köhnlein et al have conducted a study in 2014 with the intention to assess survival in chronic hypercapnic COPD patients using NPPV in addition to standard treatment for at least 6 hours at night and anytime during daytime. The results provided evidence that NPPV addition in a group of stable COPD patients reduces hypercapnia, improves overall survival, exercise capacity and HRQoL over 1 year when comparing with guideline-oriented COPD treatment without NPPV [18]. In the study conducted by Raveling et al in 2018, NIV was initiated in COPD patients in a stable condition and after an episode of acute respiratory failure using BiPAP ventilators [6]. Compliance to the ventilator after 3 months was $6.6 \pm 2.0$ hours per night and $80 \%$ of the patients have used NIV for more than 5 hours per night. A higher body mass index and forced expiratory volume in one second, a lower bicarbonate before NIV initiation, younger age and NIV initiated in stable conditions were independently associated with better survival [6].

In high-pressures, NIV may develop a haemodynamic compromise due to reduced venous return from high intra-thoracic pressures. These factors may affect results of NIV in NIV-naïve patients or in those with compromised cardiac performance [19]. Still, as shown by Dreher et al, high intensity NPPV using a controlled mode of ventilation with a mean inspiratory pressure of $29 \mathrm{mBar}$ is well tolerated by COPD patients with hypercapnic respiratory failure. Hence, high-intensity NPPV is superior 
to low-intensity NPPV using an inspiratory pressure of $15 \mathrm{mBAR}$ in controlling nocturnal hypoventilation in this population of patients [21]. It is also advantageous in improving dyspnoea during physical activity, lung function and HRQoL [21]. They have been reported two disadvantages of high-intensity NPPV: patients need more days in hospital to acclimatise and there is an increased expiratory leakage comparing to low-intensity NPPV [21].

\section{Types of non-invasive ventilators}

\section{Negative airway pressure devices}

Techniques to deliver ventilatory support have developed in $19^{\text {th }}$ century and became popular during the polio epidemic in early $20^{\text {th }}$ century [8]. There are two types of negative pressure ventilation (NPV). One is tank ventilation that provides intermittent sub-atmospheric pressure around the whole body. The other one is cuirass ventilator that provides negative pressure around the chest only and creates a gradient pressure between thorax and lower body, which may increase intrathoracic venous return, right cardiac output and lung perfusion [22]. Breathing pattern undergoing cuirass ventilator is a real approximation of the normal physiological breathing, with more natural distribution of air in the lungs. Hence, NPV does not restrict the patients' activities and they can be more comfortable [22].

\section{Positive airway pressure devices}

Positive airway pressure (PAP) devices offer today a consistent solution; they unload respiratory system and increase its capacity, with a consecutive reduction in neural respiratory drive and breathlessness [8]. Limitation of negative pressure devices promoted development of PAP devices to deliver continuous positive airway pressure (CPAP) and bilevel pressure support. CPAP delivers a fixed level of positive airway pressure during the whole respiratory cycle [8].

Bi-level pressure support, known as NIV, delivers a positive pressure during expiration and a higher positive pressure during inspiration to support inspiratory effort. PAP devices deliver respiratory support through oronasal or nasal mask interfaces, and not through endotracheal tube or tracheostomy, thus being considered as non-invasive devices [8]. PAP devices have well established benefits in acute and chronic respiratory failure in a large range of conditions, including COPD, obstructive sleep apnoea, obesity-related respiratory failure, progressive neuromuscular disease (NMD) and cardiogenic pulmonary oedema, through restoring respiratory muscles load-capacity balance, promoting alveolar ventilation and improving gas exchange [8].

\section{Non-invasive ventilation act as an adjunct to pulmonary rehabilitation}

NIV may be used as an adjunctive therapy to PR that unloads the respiratory muscles with the aim to increase the intensity of exercise training in selected patients with severe chronic respiratory disease who have a suboptimal response to exercise [1]. The benefits appear to be more marked in patients with severe COPD, and higher tolerated positive pressure may lead to greater improvements [1], with improved exercise performance and reduced breathlessness [23]. COPD is characterized by recurrent exacerbations leading to episodes of severe clinical deterioration requiring hospitalization and ventilatory support. Persistent hypercapnia after an episode of AECOPD is associated with excess mortality and early hospitalization [24]. Non-invasive positive airway pressure (PAP) interventions, applied during exercise, at rest and in the end-of-life setting, can be used to restore the balance of respiratory muscle load and capacity, with reducing neural respiratory drive and dyspnoea [8].

Long-term oxygen therapy (LTOT) and non-invasive ventilation (NIV) are potentially valuable therapeutic options, especially in COPD patients with severe lung hyperinflation and exercise-induced desaturation noticed during exercise training as part of a comprehensive PR programme [16]. For patients with COPD and chronic hypoxia LTOT is crucial in terms of improving survival. In these cases, use of supplemental oxygen during exercise may be associated with reduced exertional $\mathrm{SpO} 2$ and increased exercise performance [25]. The addition of nasal positive pressure ventilation to LTOT in hypercapnic patients has been shown to improve arterial blood gases, dyspnoea, quality of life and survival [20, 23]. Oxygen saturation measured by pulse oximetry ( $\mathrm{SpO} 2)$ should be $>88 \%$ during exercise; if $\mathrm{SpO} 2$ is $\leq 88 \%$ while breathing room air, supplemental oxygen should be used to maintain $\mathrm{SpO} 2$ at $>88 \%[13,26]$ or $>90 \%$ according to other authors $[22,27]$.

\section{In AECOPD}

COPD exacerbations are known to deteriorate life quality, to enhance disease progression and increase mortality [12]. Acute respiratory failure leading to acute or acute-on-chronic respiratory acidosis, develops when the respiratory muscles fall to achieve adequate alveolar ventilation despite high levels of diaphragmatic activity [28] and when appear alterations in central ventilatory control [18]. The official European Respiratory Society/American Thoracic Society clinical practical guidelines suggest to consider bi-level NIV in patients with AECOPD in three clinical settings: to prevent acute respiratory acidosis; to prevent endotracheal intubation and invasive mechanical ventilation in patients with mild to moderate acidosis and respiratory distress; and as an alternative to invasive ventilation in patients with severe acidosis and more severe respiratory distress [28]. Bi-level NIV is known to improve related symptoms, and to reduce the length of hospital stay, intubation rate and mortality rate $[11,28]$. NPPV is a life support intervention that does not require sedation, allowing the patients to communicate with the family and caregivers during the interruptions, to eat, to drink and to take decisions regarding their care. The outcomes were more favourable in COPD patients with strong cough and awake [29, 30].

The British Thoracic Society recommends the initiation of early PR within 1 month of hospital discharge after exacerbation, consisting of a minimum of twice a week supervised session, lasting between 6 to 12 weeks [17]. PR can be delivered late post-exacerbation that is 6 months after COPD exacerbations, the majority flare ups requiring hospitalisation or hospital at home services [17]. More recently, a reliable group of experts have strongly recommended the initiation of PR during and shortly after an exacerbation-related hospitalisation, as this results in clinically relevant improvements in exercise performance, lower-limb muscle function, balance and quality of life compared to usual care [31]. NIV can improve exercise tolerance with less desaturation in patients admitted to hospital with an exacerbation of chronic respiratory disease, but participation is limited in older populations; it is more suitable in younger patients with fewer comorbidities [32].

Physical and physiological recovery after a period of critical illness is slow and often incomplete, therefore limiting the period of immobility and promoting movement and exercise are attractive strategies to prevent neuromuscular weakness and enhance recovery [32, 33, and 34]. Survivors from an acute respiratory failure have exercise limitation and decreased physical quality that can persist up to 5 years after hospital discharge. These patients find difficult to lift and carry groceries, climb stairs, bend, kneel, walking moderate distance and perform other routine activities of daily living [33]. Early mobilisation and exercise therapy within the ICU target the goals of enhancing the functional outcome, health-related quality of life and reducing of health-care utilization [16].

Wright et al studied if intensive (90 min) vs standard (30 min) physical rehabilitation therapy in critically ill patients after receiving 48 hours or more of either invasive or non-invasive ventilation added any benefit at 6-month time [34]. They found no difference in either the primary 
outcome of self-reported physical health at 6 months, or the secondary outcomes like functional ability, independence, length of ICU and hospital stay [34]. Moss et al proposed a graduate manner to increase the physical training programme, for $30 \mathrm{~min}$ while in ICU up to $60 \mathrm{~min}$ when the patient was in a regular ward, in an outpatient setting or at home, with 5 components: techniques for proper breathing during exercise; progressive range of motion; therapeutic exercises addressed to the muscle strength; exercises designed to improve core mobility and strength; and functional mobility retraining including bed mobility, transfers, gait and balance [33]. Unfortunately, an intensive physical therapy programme did not improve long-term physical functioning compared with a standard-of-care physical therapy programme [33].

Airway clearance during chest infections through intensive physiotherapy consists in a modified active cycle of breathing technique accompanied by physical procedures such as percussions and shaking, and manually assisted cough; however, standard or intensive physiotherapy might be tiring for the patients and can precipitate episodic oxygen desaturation [35]. There are some cough augmentation techniques like: cough after inspiration supported by a NIV ventilator type BiPAP; exsufflationassisted cough with delivery of negative pressure initiated manually at the end of inspiration; insufflation (given manually during inspiratory phase) and exsufflation-assisted cough with delivery of the negative pressure immediately preceding the cough effort via a facial mask [35]. Mechanical insufflation-exsufflation (MIE) technique is not an alternative for secretion clearance but can be vital for expulsing secretions from central airways for patients with weak respiratory muscles and a partially preserved bulbar (laryngeal intrinsic) muscle function [36]. MIE is well tolerated and should be considered as an adjunct to manual airway clearance techniques for patients with a peak cough flow less than 160 L/min [8].

\section{In stable COPD patients}

Exercise capacity is significantly reduced in patients with COPD and chronic hypercapnia. Reduced ventilatory capacity combined with an increased ventilatory load leads to intolerable dyspnoea at low level of exercise [37]. Therefore, NIV was proposed has an adjunct to PR in patients with severe COPD in order to allow the patients to exercise at a higher training intensity and to obtain a greater effect compared to exercise training without NIV [19], with both improvement in physical functions and exercise performance [11]. A Cochrane review performed in 2014 by Menadue et al has shown that NIV during exercise training may allow COPD patients to exercise at a higher training intensity and to achieve a greater physiological training effect compared with exercise training alone or exercise training with sham NIV [38]. It is currently unknown if the demonstrated benefit of NIV during exercise training is clinically worthwhile or cost-effective [38].

NIV delivered during exercise enables patients with severe COPD to exercise at higher intensity, to increase their exercise endurance time and walking distance [8, 39]. NPPV is a feasible and beneficial tool in hospital-based PR [39], but clinical application of NIV during physical exercise is limited by time consuming NIV setup, need for specialist supervision, limited portability of devices and the ventilator model, battery duration and poor patient tolerance [8, 40]. Another recommendation is at night in patients with chronic respiratory failure, to improve their clinical status during the exercise programme, functional capacity, HRQoL and sleep quality [8, 39]. The benefits are achieved through optimization of the respiratory muscle load-capacity-drive relationship [8].

NIV prolongs endurance during exercise in COPD, but routine use is difficult. Recently, handheld, battery operated and portable NIV (pNIV) devices, providing pressure support ventilation, require the patient to inspire and expire through a mouthpiece, are intended to be applied at the end of exercise to reduce the time to return at baseline respiratory status in COPD patients, thus acting as dyspnoea-relief tools [41]. The pNIV device delivers $18 \mathrm{cmH} 2 \mathrm{O}$ inspiratory and $8 \mathrm{cmH} 2 \mathrm{O}$ expiratory pressures, and it is used only during recovery periods interspersing bouts of moderate or high-intensity physical activity [41]. A technical limitation of these devices is that the inspiratory positive airway pressure (IPAP) and expiratory positive airway pressure (EPAP) are fixed; excessive EPAP can worsen hyperinflation and circulatory limitations. The fixed pressures may be sub-optimal in some patients; therefore, the future devices should have the ability to adjust EPAP, making the pressure support more desirable and potentially automated [41]. Future studies should investigate the additive effect of oxygen supplementation to intermittent NIV support during conventional PR [41].

Less practical in daily life but providing a greater improvement in exercise tolerance are the continuous positive pressure support devices like CPAP, inspiratory pressure support including proportional assist ventilation (PAV), and non-invasive "open" ventilation (NIOV) [15, 41, 37]. All these ventilation support strategies provide continuous unloading of the respiratory muscles and reduce the work of breathing [41], thus reducing dyspnoea and enhancing exercise tolerance in COPD patients [15]. Inspiratory pressure support strategies used during exercise increase endurance, reduce dyspnoea, unload the respiratory muscles, and sustain exercise induced lactatemia for longer [42,37].

Portable CPAP devices, light-weighted and battery-powered, are particularly useful in cases with excessive dynamic airway collapse where they provide a pneumatic stent to maintain airway patency, reduce expiratory resistance and improve expiratory airflow [8]. PAV is a mode of ventilation that matches ventilator output to patient effort, allowing the patients to prolong exercises and to achieve greater improvements in exercise performance [37]. NIOV system, operating in conjunction with a portable oxygen tank, was found to decrease respiratory muscle activation and dyspnoea, and to improve cycle ergometer exercise tolerance; it is a light, wearable 1-lb ventilator, practical for facilitating activities of everyday living [43].

They have been compared the effects of high-intensity training with mouthpiece inspiratory pressure support (IPS) delivering $10 \mathrm{cmH} 2 \mathrm{O}$ with $5 \mathrm{cmH} 2 \mathrm{O}$. IPS 10 resulted in significantly larger improvements in exercise endurance than training with IPS5 in moderate-to-severe COPD patients. Therefore, it is suggested that IPS10 may be considered as adjunct during high-intensity training through better unloading the inspiratory muscles during exercise [44]. The authors found from experience that a mouthpiece is the most practical interface for the application of noninvasive ventilatory support during exercise, due to a reduced leakage of air compared with face masks and a better tolerance by the patients [44].

Some centres use Assist Pressure Control Ventilation (APCV) mode with a high backup rate, intending to achieve controlled ventilation. The use of APCV mode during exercise implies to best set up a minimum mandatory backup rate along with a fixed inspiratory time for both patient and machine-triggered breaths. The aim is to provide optimal respiratory muscle unloading and gas exchange during exercise. There is the possibility to worsen the dynamic hyperinflation if inspiratory time is set inappropriately long, particularly if the exercise spontaneous respiratory rate increases during the exercise period [19].

Negative pressure ventilation, when used as an adjuvant to PR, improves lung function, increases exercise capacity, prolongs survival and reduces exacerbations in COPD patients with exercise desaturation, who have an increased mortality risk compared with non-desaturating COPD patients. The NPV group had a slower yearly decline in lung function and in 6minute walking distance, irrespective with exercise desaturation [10]. Maintenance of NPV reduces long-term mortality in COPD patients, irrespective of the presence of desaturation during the 6MWT. Huang et 
al [22] initiated an unsupervised home endurance exercise programme and patients underwent once a week a comprehensive hospital-based PR programme where they received NPV via the cuirass ventilatory settings for $60 \mathrm{~min}$. The authors have shown that NPV can improve the walking distance and reduce the yearly decline of lung function, exacerbations and hospitalization rates, and medical costs in patients with COPD during a 5-year observation [22]. It is speculated that reduction of lung function decline might be a consequence of NPV effects in improving ventilation pattern, arterial blood gas exchange and unloading of inspiratory muscles, thus reducing the work of breathing in COPD patients; conclusively, NPV may be used as an adjunctive therapy to PR [22]. Another benefit of NPV could be an improvement in clearance secretions and inflammation and decrease in AECOPD rate [22].

Recently, the same group of researchers have shown that a programme of maintenance NPV for COPD patients suggests that beneficial clinical effects are produced by decreasing dead space and inhomogeneous lung ventilation, increasing pulmonary perfusion and clearance of mucus plugging that is associated with lung atelectasis, thus leading to improved ventilation-perfusion mismatching and shunting, and reduced hypoxaemia at rest and during exercise [10]. The NPV programme included NPV support, breathing training and an educational programme (relaxation techniques and a home pacing walking exercise) in daily clinical practice; breathing training consisted of pursued-lipped, controlled and diaphragmatic breathing [22].

\section{At home}

After successfully completing a course of PR, the only way to maintain the achieved improvements is to continue with a home-based exercise programme and participate to follow-up visits in the PR centre. The scope is to make chronic respiratory patients to become more active in daily living life and to preserve endurance capacity, psychological and cognitive benefits [12]. NIV can be used for long-term treatment of chronic respiratory failure at home. High-pressure NIV is addressed to the patients who have persistent hypercapnia for 2 to 4 weeks after resolution of respiratory acidemia that has required acute NIV [24]. In COPD patients, long-term NIV decreases arterial partial pressure of carbon dioxide $(\mathrm{PaCO} 2)$ and improves mortality [16]. Effective NIV, that significantly reduces elevated $\mathrm{PaCO} 2$, is well tolerated and associated with improvements of quality of life and long-term survival [11]. After 12 weeks of use at home the pNIV devices, patients reported reduced anxiety and recovery time from breathlessness, as well as improvement in the speed, duration and confidence to undertake activities of daily living [41].

Another treatment option would be the use of NIV in addition to oxygen therapy in home setting, at least for 6 hours per night. Recent results of a randomized clinical trial published in 2017 support the use of home oxygen therapy and home mechanical ventilation (HOT-HMV) in COPD patients following acute life-threatening exacerbation of COPD, with acute respiratory failure and persistent hypercapnia. In HOT-HMV clinical trial, the addition of home NIV to home oxygen therapy has prolonged the time to readmission or death within 12 months from 1.4 to months to 4.3 months [24]. Patients with severe COPD receiving HMV can easily use NPPV during walking without changing ventilator settings or equipment [45]. When using only oxygen supplementation, arterial oxygen tension decreases during walking, but it increases when NPPV is used in addition to supplemental oxygen, with less dyspnoea and increased walking distance [45]. Moreover, NPPV used during exertion may prevent death from hypoxia-induced complications, especially arrhythmias [45].

\section{In palliative care services}

In advanced chronic patients admitted to palliative care services, it is essential to reduce breathlessness and improve survival, quality of life and function. The use of PAP devices in end-stage hypercapnic disease is frequently implemented in clinical practise, as suggested by international guidelines. Notably, timing of its initiation remains controversial because of potential side-effects as mask discomfort and limited communication with caregivers and family [8]. Although studies evaluating the effects of NIV over dyspnoea when used in palliative care settings are missing, prospective longitudinal studies have shown that hospital survival amongst patients with COPD and congestive cardiac failure with do-notintubate order are unexpected high (30-60\%) [8]. Moreover, their 3month quality of life is equivalent to patients managed with acute NIV with no pre-set ceiling of care [8]. NPPV is a mean of potentially ensuring the highest quality of life during the final hours [29, 30], allowing to reduce the dose of morphine necessary to palliate dyspnoea, maintaining better cognitive function and with a similar rate of acceptance by patients compared with oxygen therapy [28]. The international guidelines suggest offering NIV to dyspnoeic patients for palliation in the setting of terminal cancer or other terminal conditions [28].

\section{Conclusions}

Pulmonary rehabilitation is a unique non-pharmacological therapy addressed to symptomatic COPD and non-COPD patients with a poor HRQoL due to breathlessness and fatigue. Because of dyspnoea, patients become more socially isolated and finally housebound. PR, LTOT and NIV bring hope in this difficult and long-term fight with a chronic respiratory disease, encouraging patients to meet other people with same disability and fear from illness and death, to exercise together and to continue their lives in a better condition. There is enough room, need and available technology to implement NIV worldwide, to encourage both patients and researchers to look ahead and find the best answers for the reported practical problems.

\section{References}

1. Spruit MA, Singh SJ, Garvey C, et al. (2013) An official American Thoracic Society/European Respiratory Society statement: Key concepts and advances in pulmonary rehabilitation. Am J Respir Crit Care Med, 188(8):13-64.

2. Houben-Wilke S, Augustin IM, Vercoulen JH, et al. (2013) COPD stands for complex obstructive pulmonary disease. Eur Respir Rev 2018; 27:180027.

3. Ciobanu LD. What does it mean to be physically active in chronic respiratory diseases? ECPRM. 5.2:135-139.

4. Spruit MA, Wouters EFM. (2019) Organizational aspects of pulmonary rehabilitation in chronic respiratory diseases. Respirology.

5. Sandoz JS, Roberts MM, Cho JG, et al. (2017) Magnitude of exercise capacity and quality of life improvement following repeat pulmonary rehabilitation in patients with COPD. Int $J$ Chron Obstruct Pulmon Dis. 12:1085-1091.

6. Raveling T, Bladder G, Vonk JM, et al. (2018) Improvement in hypercapnia does not predict survival in COPD patients on chronic noninvasive ventilation. Int J COPD. 13:3625-3634.

7. Koolen EH, van Hees HW, van Lummel RC, et al. (2019) "Can do" versus "do do": a novel concept to better understand physical functioning in patients with chronic obstructive pulmonary disease. J Clin Med. 8:340.

8. D'Cruz RF, Kaltsakas G, Suh ES, et al. (2018) Positive airway pressure devices for the management of breathlessness. Curr Opin Support Palliat Care. 12:246-252.

9. Neder JA, Marillier M, Bernard AC, et al. (2019) The integrative physiology of exercise training in patients with COPD. COPD. 16:1-14.

10. Huang HY, Lo CY, Yang LY, et al. (2019) Maintenance negative pressure ventilation improves survival in COPD patients with exercise desaturation. J Clin Med. 8: 562. 
11. Jung JH, Lim YH, Lee JW, et al. (2018) Remarkable improvement in a very severe chronic obstructive pulmonary disorder patient after use of noninvasive intermittent positive pressure ventilator. A case report. Medicine (Baltimore). 97:43(e12877).

12. Candemir I. (2018) Advances in pulmonary rehabilitation for chronic obstructive pulmonary disease and associated conditions. In: COPD - an update in pathogenesis and clinical management. Intech Open: Cormac McCarthy, 131-151.

13. Garvey C, Bayles MP, Hamm LF, et al. (2016) Pulmonary rehabilitation exercise prescription in chronic obstructive pulmonary disease: review of selected guidelines. An official statement from the American Association of Cardiovascular and Pulmonary Association. J Cardiopulm Rehabil Prev. 36:75-83.

14. Güell MR, Cejudo P, Ortega F, et al. (2017) Benefits of longterm pulmonary rehabilitation maintenance program in patients with severe chronic obstructive pulmonary disease. Three-year follow-up. Am J Respir Crit Care Med. 195(5):622-629.

15. Ambrosino N. (2006) Assisted ventilation as an aid to exercise training: a mechanical doping? Eur Respir J, 27:3-5.

16. Spruit MA, Rochester CL, Pitta F, et al. (2017) Pulmonary rehabilitation, physical activity, respiratory failure, and palliative respiratory care. Thorax, 74(7):693-699.

17. Bolton CE, Bevan-Smith EF, Blakey JD, et al. (2013) British Thoracic Society guideline on pulmonary rehabilitation in adults. Thorax. 68:ii1-ii30.

18. Köhnlein T, Windisch W, Köhler D, et al. (2014) Non-invasive positive pressure ventilation for the treatment of severe stable chronic obstructive pulmonary disease: a prospective, multicentre, randomised, controlled clinical trial. Lancet Respir Med, 2: 698-705.

19. Menadue C, Piper AJ. (2019) Pressuring stable patients with hypercapnic COPD to exercise. Respirology, 24:195-196.

20. Liao H, Pei W, Li H, et al. (2017) Efficacy of long-term noninvasive positive pressure ventilation in stable hypercapnic COPD patients with respiratory failure: a meta-analysis of randomized controlled trials. Int J Chron Obstruct Pulmon Dis, 12: 2977-2985.

21. Dreher M, Storre JH, Schmoor C, et al. (2010) High-intensity versus low-intensity non-invasive ventilation in patients with stable hypercapnic COPD: a randomised crossover trial. Thorax, 65(4):303-308.

22. Huang HY, Chou PC, Joa WC, et al. (2016) Pulmonary rehabilitation coupled with negative pressure ventilation decreases decline in lung function, hospitalizations, and medical cost in COPD. Medicine (Baltimore), 95(41):e5119.

23. Garrod R, Mikelsons C, Paul EA, et al. (2000) Randomized controlled trial of domiciliary noninvasive positive pressure ventilation and physical training in severe chronic obstructive pulmonary disease. Am J Respir Crit Care Med, 162:1335-1341.

24. Murphy PB, Rehal S, Arbane G, et al. (2017) Effect of home noninvasive ventilation with oxygen therapy vs oxygen therapy alone on hospital readmission or death after an acute COPD exacerbation. JAMA, 317(21): 2177-2186.

25. Gloeckl R, Osadnik C, Bies L, et al. (2019) Comparison of continuous flow versus demand oxygen delivery systems in patients with COPD. A systematic review and meta-analysis. Respirology, 24:329-337.

26. Karim HMR, Burns KEA, Ciobanu LD, et al. (2019) Noninvasive ventilation: education and training. A narrative analysis and an international consensus document. Adv Respir Med, 87:37-45.
27. Duiverman ML, Wempe JB, Bladder G, et al. (2011) Two-year home-based nocturnal noninvasive ventilation added to rehabilitation in chronic obstructive pulmonary disease patients: a randomized control trial. Respir Res, 12:112.

28. Rockwerg B, Brochard L, Elliott MW, et al. (2017) Official ERS/ATS clinical practice guidelines: noninvasive ventilation for acute respiratory failure. Eur Respir J, 50:1602426.

29. Levy M, Tanios MA, Nelson D, et al. (2004) Outcomes of patients with do-not-intubate orders treated with noninvasive ventilation. Crit Care Med, 32(10):2002-2007.

30. Schettino G, Altobelli N, Kacmarek RM. (2005) Noninvasive positive pressure ventilation reverses acute respiratory failure in select "do-not-intubate" patients. Crit Care Med, 33(9):19761982.

31. Spruit MA, Singh SJ, Rochester CL, et al. (2018) Pulmonary rehabilitation for patients with COPD during and after an exacerbation-related hospitalisation: back to the future? Eur Respir J, 51:1701312.

32. Dyer F, Flude L, Bazari F, et al. (2011) Non-invasive ventilation (NIV) as an aid to rehabilitation in acute respiratory disease. BMC Pulm Med, 11:58.

33. Moss M, Nordon-Craft A, Malone D, et al. (2016) A randomised trial of an intensive physical therapy program for patients with acute respiratory failure. Am J Respir Crit Care Med, 193(10): 1101-1110.

34. Wright SE, Thomas K, Watson G, et al. (2018) Intensive versus standard physical rehabilitation therapy in the critically ill (EPICC): a multicentre, parallel- group, randomised controlled trial. Thorax, 73:213-221.

35. Chatwin M, Ross E, Hart N, et al. (2003) Cough augmentation with mechanical insufflation/exsufflation in patients with neuromuscular weakness. Eur Respir J, 21:502-508.

36. Bach JR. (2003) Mechanical insufflation/exsufflation: has it come of age? A commentary. Eur Respir J, 21:385-386.

37. Hawkins P, Johnson LC, Nikoletou D, et al. (2002) Proportional assist ventilation as an aid to exercise training in severe chronic obstructive pulmonary disease. Thorax, 57:853-859.

38. Menadue C, Piper AJ, van't Hul AJ, et al. (2014) Non-invasive ventilation during exercise training for people with chronic obstructive pulmonary disease (review). Cochrane Database Syst Rev, 5:CD007714.

39. Köhnlein T, Schönheit-Kenn U, Winterkamp S, et al. (2009) Noninvasive ventilation in pulmonary rehabilitation of COPD patients. Respir Med, 103(9):1329-1336.

40. Vitacca M, Kaymaz D, Lanini B, et al. (2018) Non-invasive ventilation during cycle exercise training in patients with chronic respiratory failure on long-term ventilatory support: a randomized controlled trial. Respirology, 23: 182-189.

41. Vogiatzis I, Chynkiamis N, Armstrong M, et al. (2019) Intermittent use of portable NIV increases exercise tolerance in COPD: a randomized, cross-over trial. J Clin Med, 8:94.

42. Polkey MI, Hawkins P, Kyroussis D, et al. (2000) Inspiratory pressure support prolongs exercise induced lactataemia in severe COPD. Thorax, 55(7):547-549.

43. Porszasz J, Cao R, Morishige R, et al. (2013) Physiologic effect of an ambulatory ventilation system in chronic obstructive pulmonary disease. Am J Respir Crit Care Med, 188(3):334-342.

44. van't Hul A, Gosselink R, Hollander P, et al. (2004) Acute effects of inspiratory pressure support during exercise in patients with COPD. Eur Respir J, 23:34-40.

45. Dreher M, Storre JH, Windisch W. (2007) Noninvasive ventilation during walking in patients with severe COPD: a randomised cross-over trial. Eur Respir J, 29:930-936. 